\title{
Diamond Bragg superlattice grown in microwave gas discharge for obtaining photoluminescence of single diamond color centers comprising a dense 3D ensemble
}

\author{
V.A. Kukushkin $^{1,2}$, M.A. Lobaev ${ }^{1}$, D.B. Radischev ${ }^{1}$, S.A. Bogdanov ${ }^{1}$, M.N. Drozdov ${ }^{3}$, \\ V.A. Isaev ${ }^{1}$, A.L. Vikharev ${ }^{1}$, and A.M. Gorbachev ${ }^{1,2}$ \\ ${ }^{1}$ Institute of Applied Physics of the Russian Academy of Science, Nizhny Novgorod, Russia, \\ vakuk@appl.sci-nnov.ru \\ ${ }^{2}$ Nizhny Novgorod State University named after N.I. Lobachevsky, Nizhny Novgorod, Russia \\ ${ }^{3}$ Institute for Physics of Microstructures of the Russian Academy of Sciences, Nizhny Novgorod Region, Russia
}

Due to its unique qualities (radiation and chemical endurance, high thermal conductivity, a very large breakdown electric field and others) chemically vapor deposited (CVD) diamond is a promissing material for modern photonics and optoelectronics. Traditional optical devices on CVD diamond (e.g. waveguides and photonic crystals) are based on its post-growth treatment, for example on the creation of a periodic pattern in a CVD diamond film by electronic beam lithography, focused ion beam milling or a laser beam ablation [1]. However, such technologies have intrinsic limitations connected with difficulty to achieve a desirable spatial resolution, problems of reproducibility, defects and the roughness of the structure interfaces resulting in undesirable photon scattering.

Therefore, it seems promising to form a CVD diamond optical devices (for example, Bragg superlattices) directly during the growth process to ensure their atomscale precision and quality. But this way encounters a difficulty that there is no heteropair for diamond (i.e. a substance with crystal lattice parameters very close to those of diamond, but a different refraction index). In principle, there exists difference of the refraction indices of diamond isotopes ${ }^{12} \mathrm{C}$ and ${ }^{13} \mathrm{C}$, which, therefore, could be employed to form structures for CVD diamond optical devices. But this difference is very small (of order $10^{-3}$ and smaller in the visible and nearinfrared wavelength region). This means that for obtaining a highly reflective Bragg superlattice it is necessary to grow a very large (several thousand) number of alternating layers of ${ }^{12} \mathrm{C}$ and ${ }^{13} \mathrm{C}$ with very precisely repeated parameters, which is technologically impossible now.

So, nowadays the only way to create a highly reflective CVD diamond Bragg superlattice is the modulation doping method consisting in the growth of interchanging highly and low doped CVD diamond layers $[2,3]$ (please see fig. 1). The oscillations of free charge carriers (holes) in alternating electromagnetic field in the highly doped layers cause the change of the dielectric permittivity of these layers in comparison with the dielectric permittivity of the adjacent low doped layers. As a result, dielectric permittivity becomes periodically modulated in space. It is well-known that for certain wavelengths satisfying the Bragg condition electromagnetic radiation is efficiently reflected from such a superlattice even in the case of a small relative difference of the dielectric permittivities of the highly and low doped layers if the number of these layers is sufficiently large. This effect is due to coherent summation (i.e., constructive interference) of waves reflected from the consecutive boundaries of the layers.

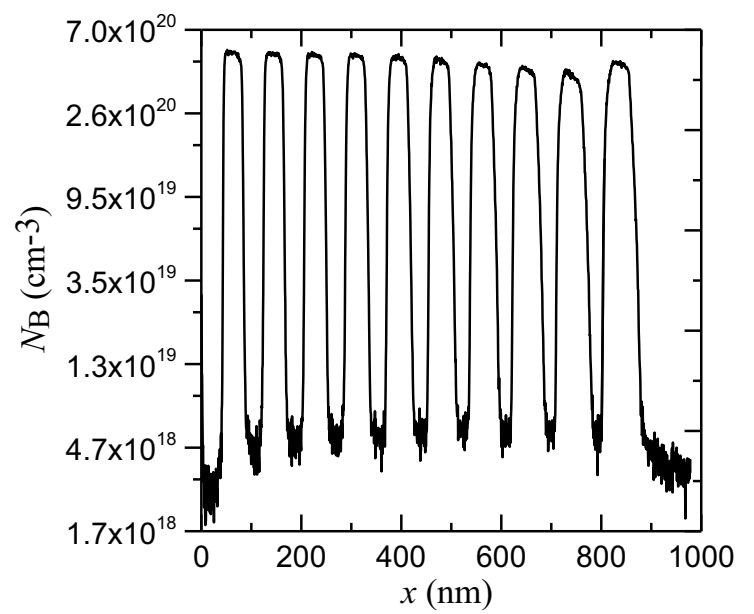

Fig 1. Secondary ion mass spectrometry (SIMS) measurements of dopant (boron) atom concentration $N_{B}$ as a function of the distance $x$ from the boundary of the CVD diamond film with air

We have grown such a CVD diamond Bragg superlattice on a home-built microwave gas discharge reactor [4]. The reactor consists of a cylindrical microwave cavity with a quartz tube placed inside on the axis of the cavity. Microwave radiation with an input power of $1.5 \mathrm{~kW}$ and a frequency of $2.45 \mathrm{GHz}$ generates a plasma at a gas pressure 40 Torr just above a substrate holder. A non-vortex laminar gas flow with the total flow rate of $900 \mathrm{sccm}$ inside the quartz tube is employed for a quick change of a gas mixture within the reactor. The low boron doped CVD diamond layers were grown in the feed gas mixture $\mathrm{H}_{2}+\mathrm{CH}_{4}\left(\mathrm{CH}_{4} / \mathrm{H}_{2}=0.1 \%\right)$. This gas mixture was quickly (in comparison with characteristic growth time) changed to $\mathrm{H}_{2}+\mathrm{CH}_{4}+\mathrm{B}_{2} \mathrm{H}_{6}$ (the same methane content, $\mathrm{B}_{2} \mathrm{H}_{6} / \mathrm{H}_{2}=0.1 \%$ ) with an electronic switch for growing the highly boron-doped CVD diamond layers. A time value for achieving the gas mixture change in the reactor (i.e., the reactor residence time) is estimated by the Ar emission line dynamics and is approximately equal to $5 \mathrm{~s}$. Due to the presence of residual boron in the reactor the low boron doped CVD diamond layers possessed some small boron concentration. Hydrogen sulfide (added to the gas mixture during the growth of the low boron doped layers) was used as a chemical getter to decrease the residual boron contamination.

Type IIa high pressure high temperature (HPHT) diamond substrates from New Diamond Technologies with dimensions $3.5 \times 3.5 \times 0.5 \mathrm{~mm}$ were used for the CVD diamond boron-doped Bragg superlattice growth. 
The substrates were pre-treated by inductive coupled plasma in order to get rid of defects introduced during the polishing procedure. As a result, surface roughness $R_{\mathrm{a}} \approx 0.1 \div 0.3 \mathrm{~nm}$ was achieved.

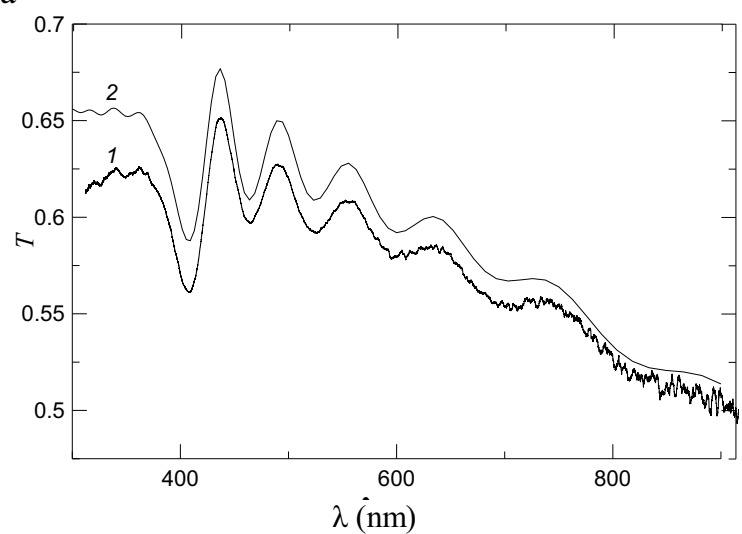

Fig. 2. Power transition coefficient $T$ as a function of the radiation vacuum wavelength $\lambda$ for normal incidence. Curve $1-$ experimental data, 2 - calculation results

The vacuum wavelength dependence of a power transition coefficient $T$ of optical and near infrared electromagnetic radiation through the diamond sample with the CVD Bragg superlattice was measured by means of Horiba Jobin Yvon FHR-1000 monochromator under the sample irradiation from the superlattice (i.e. top) side by a stabilized light source along the normal to its surface (fig. 2). The obtained experimental data were compared with the results of simulations by means of the transfer matrix method. We considered the coherence length of the used light to be larger than the superlattice thickness and, therefore, took into account the interference of fields of the waves reflected from the boundaries of the layers comprising the superlattice. In the same time, we assumed that this coherence length was smaller than the thickness of the HPHT diamond substrate (approximately $0.5 \mathrm{~mm}$ ). So, we summed not the fields, but the intensities of the waves reflected from the interfaces the Bragg superlattice - the HPHT diamond substrate and the HPHT diamond substrate - air. Experimental curve 1 position slightly below theoretical curve 2 is explained by some additional absorption not taken into account in the calculations and connected with the sample contamination.

The Bragg CVD diamond superlattice can be used to significantly increase the spatial resolution of the color center photoluminescence collection at a fixed exciting laser beam focal volume. It will allow to collect the photoluminescence of individual color centers in their dense $3 \mathrm{D}$ ensembles, which is needed for quantum computing and memory applications and for measurement and imaging with high spatial resolution. The suggested method is based on the creation of a narrow waveguide for the color center photoluminescence with a small number of allowed vertical indices of guided modes. The waveguide is formed between the top surface of a CVD diamond film and an underlaid mirror - the Bragg superlattice due to the total internal reflection from the former and the Bragg reflection from the latter. Then, the frustrated total internal reflection (FTIR) method is employed to extract through the top surface of the CVD diamond film the color center photoluminescence contained only in guided modes with the same vertical index. Color centers which lie near the minima of the electric field depth distribution of these modes in the CVD diamond Bragg superlattice give small contribution to the FTIR-extracted photoluminescence and, therefore, can be disregarded. So, the exciting laser beam focal volume is divided into "dark" (i.e. lying at the minima of these modes electric field depth distribution) and "bright" (i.e. lying at the maxima of these modes electric field depth distribution) layers. In consequence, an effective (with respect to the excitation of the FTIR-extracted modes of the color center photoluminescence) exciting laser beam focal volume is comprised by only "bright" layers and, therefore, is smaller than the whole exciting laser beam focal volume. So, the maximal value of the color center $3 \mathrm{D}$ concentration still compatible with obtaining single color center photoluminescence becomes of order the inverse effective focal volume, which is larger than the inverse focal volume, i.e. its value in the Bragg superlattice absence.

According to the results of simulation made for a case when color centers are NV centers, the suggested method allows to increase the maximal value of the $\mathrm{NV}$ center concentration still compatible with obtaining single color center photoluminescence by several times at a fixed exciting laser beam focal volume. The increase is provided without the deterioration of the NV center photoluminescence collection efficiency.

The authors are grateful to P. A. Yunin for the processing of the SIMS method data. The work was supported by the Russian Science Foundation (project No. 16-19-00163).

\section{References}

1. Tomljenovic-Hanic, S., Karle, T. J., Greentree, A. D., Gibson, B. C., Fairchild, B. A., Stacey, A., and Prawer, S. Diamond-Based Optical Waveguides, Cavities, and Other Microstructures // Optical Engineering of Diamond, edited by R. P. Mildren and J. R. Rabeau. Weinheim, Germany: Wiley-VCH Verlag \& Co. KGaA. 2013. P. 311-351.

2. Fiori, A., Bousquet, J., Eon, D., Omnès, F., BelletAmalric, E., and Bustarret, E. Boron-doped superlattices and Bragg mirrors in diamond // Appl. Phys. Lett. 2014. V. 105, No. 8. Art. No. 081109 (1-4).

3. Kukushkin, V. A., Lobaev, M. A., Radischev, D. B., Bogdanov, S. A., Drozdov, M. N., Isaev V. A., Vikharev, A. L., and Gorbachev, A. M. Bragg superlattices formed in growing chemically vapor deposited diamond // Journal of Applied Physics. 2016. V. 120, No. 22. Art. No. 224901 (1-5).

4. Vikharev, A. L., Gorbachev, A. M., Lobaev, M. A., Muchnikov, A. B., Radishev D. B., Isaev, V. A., Chernov, V. V., Bogdanov, S. A., Drozdov, M. N., and Butler, J. E. Novel microwave plasma-assisted CVD reactor for diamond delta doping // Physica Status Solidi Rapid Research Letters. 2016. V. 10, No. 4. P. 324-327. 